\title{
Preparation, Characterization, and Antitumor Evaluation of Electrospun Resveratrol Loaded Nanofibers
}

\author{
Hui Zhou, ${ }^{1}$ Xiaoxing Liu, ${ }^{2}$ Fenglei $W u,{ }^{3}$ Junting Zhang, ${ }^{4}$ Zhen $W u,{ }^{4}$ \\ Haitao Yin, ${ }^{5}$ and Hui Shi ${ }^{1}$ \\ ${ }^{1}$ Department of Neurosurgery, Affiliated Lianyungang Hospital of Xuzhou Medical College, Lianyungang 222000, China \\ ${ }^{2}$ Department of Neurosurgery, Jiangsu Taizhou People's Hospital, Taizhou 225300, China \\ ${ }^{3}$ Department of Oncology, Affiliated Lianyungang Hospital of Xuzhou Medical College, Lianyungang 222000, China \\ ${ }^{4}$ Department of Neurosurgery, Beijing Tiantan Hospital, Capital Medical University, Beijing 100050, China \\ ${ }^{5}$ Department of Radiotherapy, The Central Hospital of Xuzhou, Affiliated Hospital of Southeast University, Xuzhou 221009, China
}

Correspondence should be addressed to Haitao Yin; haitaoyin@xzmc.edu.cn and Hui Shi; shihuilyg@163.com

Received 18 January 2016; Accepted 12 April 2016

Academic Editor: Martin Andersson

Copyright (C) 2016 Hui Zhou et al. This is an open access article distributed under the Creative Commons Attribution License, which permits unrestricted use, distribution, and reproduction in any medium, provided the original work is properly cited.

Resveratrol has been reported as a potential antitumor agent for several years. The possible application was greatly hampered by its poor solubility. The current study reported the construction of electrospun Resveratrol loaded nanofibers with methoxypolyethylene glycol-poly(caprolactone) (mPEG-PCL) block copolymers as drug carriers. Characterization studies showed the successful encapsulation of Rsv in the nanofibers with an in vitro sustained release pattern. In vitro XTT assay suggested the superior cytotoxicity of Rsv-NFs with more apoptosis induction on cancer cells. Cells that exposed to Rsv-NFs showed less clonogenic ability when compared to the equivalent dose of free Rsv. In addition, the migration and invasion ability of cells were also significantly lower when treated with Rsv-NFs. In the in vivo study, local implantation of Rsv-NFs greatly increased the growth inhibitory effect compared to free Rsv. Therefore, results from the current study demonstrated a promising way to improve the antitumor effect of Rsv by nanofiber delivery.

\section{Introduction}

Chinese herbal medicine, also known as Traditional Chinese Medicine, has attracted increasing interest in recent years. There are plenty of studies demonstrating the potential anti-inflammation, antitumor, antioxidant effects of herbal medicines such as Tetrandrine, Curcumin, Resveratrol, and Harmine [1-4]. Recently more and more researchers focus on the potential anticancer effect of herbal medicines as well as their possible mechanisms [5-7].

Among these herbal medicines, Resveratrol (Rsv) attracts intensive interest for its potential in cancer therapy. Previous studies have demonstrated the inhibitory effect of Rsv against a series of cancers, including glioma, colon cancer, and gastric cancer $[8,9]$. It is reported that Rsv could induce apoptosis through regulating apoptosis-related proteins [10]. Moreover, Rsv could also block several pro-proliferative pathways, such as PI3k/Akt/mTOR and MAPK pathways [11, 12]. In addition, there are some reports stating that Rsv could enhance the antitumor effect of commonly used chemotherapeutics, which makes it a promising chemotherapy adjuvant $[13,14]$.

One hindrance for the application of Rsv is its poor solubility. However, the hydrophobicity of Rsv renders it a good model drug for nanodelivery systems. In previous studies, we prepared Rsv-loaded nanoparticles (Rsv-NPs) with amphiphilic polymers as drug carriers $[2,15,16]$. Characterization of Rsv-NPs showed good aqueous stability and loading efficiency and controlled release kinetics. In vitro studies indicated the superior cell inhibitory effect over free Rsv. In the in vivo study, Rsv-NPs showed better antitumor effect due to the EPR (Enhanced Permeability and Retention) effect. Nevertheless, there are still some disadvantages, such as lack of targeted ability and low loading efficiency.

To overcome these disadvantages, drug loaded nanofibers based on amphiphilic copolymers become an alternative to 
deliver the drugs. There are some studies focusing on the construction of drug loaded nanofibers with herbal medicine as model drugs $[17,18]$. Drug loaded nanofibers have a large surface area-to-volume ratio, which enables an excellent drug loading efficiency. Moreover, previous studies focusing on the shape effect of nanostructures have demonstrated that nanofibers showed 10 times circulation times longer than spherical nanostructures, thereby exhibiting stronger tumor shrinkage [19].

In the current study, we prepared Rsv-loaded nanofibers (Rsv-NFs) with PCL-PEG as drug carriers by electrospinning. Rsv-NFs were characterized by DLS, Uvis, and FTIR. In vitro studies were performed including cellular uptake, cytotoxicity, migration, and invasion studies. Animal study was performed to evaluate the in vivo antitumor effect of Rsv-NFs.

\section{Materials and Methods}

2.1. Materials. Resveratrol and methoxypolyethylene glycol (mPEG) was purchased from Sigma Aldrich Co. (St. Louis, MO, USA). $\varepsilon$-Caprolactone ( $\varepsilon$-CL, Sigma) was purified by drying over $\mathrm{CaH}_{2}$ at room temperature and distillation under reduced pressure. Coumarin-6 was purchased from Sigma (St. Louis, MO, USA). All other chemicals were of analytical grade. Human glioma cell line U87 was obtained from Shanghai Institute of Cell Biology (Shanghai, China).

\subsection{Methods}

2.2.1. Synthesis of mPEG-PCL Block Copolymers. mPEGPCL block copolymers were synthesized by a ring-opening copolymerization [20]. Certain amount of CL was mixed with $\mathrm{mPEG}$ with the presence of stannous octoate $(0.1 \% \mathrm{wt} / \mathrm{wt})$ in a tube connecting to a vacuum system and then incubated in an oil bath at 130 for $48 \mathrm{~h}$. Dichloromethane (DCM) was utilized to dissolve the raw synthesized polymers and the obtained solution was precipitated into cold methanol to eliminate the excess monomer and oligomer. The precipitates were then purified by filtration before being thoroughly dried at lower pressure. The polymers were then characterized by ${ }^{1} \mathrm{H}$ NMR and gel permeation chromatography (GPC).

2.2.2. Preparation of Rsv-Loaded Nanofibers. Rsv-NFs were prepared using an electrospinning device. mPEG-PCL was dissolved in ethanol and water at a ratio of $5: 1$ to generate a $10 \%$ transparent polymer solution. Then $5 \mathrm{wt} \%, 10 \mathrm{wt} \%$, and $15 \mathrm{wt} \%$ Rsv with respect to mPEG-PCL were added and dissolved to the polymeric solution. The solution was then sonicated for $30 \mathrm{~s}$ and then loaded in a $5 \mathrm{~mL}$ syringe with a needle connected to a high voltage of DC supply. The distance between the top of the needle and the collector is $12 \mathrm{~cm}$. The flow speed of the solution was set at $0.5 \mathrm{~mL} / \mathrm{h}$. The nanofiber was collected through an aluminum foil. Coumarin- 6 loaded Rsv-NFs were prepared with the addition of coumarin-6 (1 wt \%) together with Rsv.

2.2.3. SEM, FTIR, and Loading Efficiency. Scanning Electron Microscopy (SEM) (JEOL, Japan) was performed to observe the morphology of Rsv-NFs. Fourier transform infrared
(FTIR) spectra were measured by a Perkin-Elmer Paragon 1000 Fourier transform spectrometer. Drug loading efficiency was determined by high performance liquid chromatography (HPLC, Shimadzu LC-10AD, Japan) system with a Shimadzu UV detector. Briefly, a predetermined amount of $10 \mathrm{mg}$ Rsv-NFs was dissolved in mobile phase consisting of methanol (spectral grade, Merck, Germany)/double-distilled water/glacial acetic acid $(48 / 52 / 0.05, \mathrm{v} / \mathrm{v} / \mathrm{v})$. The pump rate was $1 \mathrm{~mL} / \mathrm{min}$. The detected wavelength was $303 \mathrm{~nm}$ and the retention time was about $4.2 \mathrm{~min}$. The encapsulation efficiency was calculated as the following equation: encapsulation efficiency $(\%)=$ weight of the drug in nanofibers/weight of the feeding drugs $* 100 \%$.

2.2.4. In Vitro Release. For the in vitro release study, a small piece of $20 \mathrm{mg}$ Rsv-NFs mat was immersed in a $50 \mathrm{~mL}$ glass tube with $30 \mathrm{~mL}$ PBS containing 5\% ethanol and the tube was incubated at 37 in a thermostat shaker. At preset time points, the nanofiber mat was transferred to another tube with the same medium. The released Rsv in the previous medium buffer was extracted by the mobile phase and measured on a HPLC. The concentration of released Rsv was calculated and plotted at different time points.

2.2.5. Cellular Uptake. U87 cells were seeded in 6-well plates and allowed to grow for $24 \mathrm{~h}$. Coumarin-6 loaded Rsv-NFs were incubated with cells in 6-well plate for $2 \mathrm{~h}$. After cells were washed two times by PBS, cells were observed under a fluorescent microscopy.

For quantitative study, Res-NFs were incubated with cells for different time periods with different concentrations. Cells were then trypsinized, washed by PBS for 3 times, and centrifuged at $1500 \mathrm{rpm}$ for 5 mins. The cell pellets were dissolved in $1 \mathrm{~mL}$ methanol and sonicated for $60 \mathrm{~s}$, followed by a centrifugation of $10000 \mathrm{rpm}$ for 5 mins. Supernatant was collected and run on a U-spectrometer at a wavelength of $310 \mathrm{~nm}$.

2.2.6. In Vitro Cytotoxicity. The in vitro cytotoxicity of RsvNFs with different Rsv loadings was evaluated by XTT assay. U87 was seeded in 96-well plates with a density of around $5 * 10^{3} /$ well. Cells were allowed to adhere for $24 \mathrm{~h}$ prior to the test and then exposed to free Rsv, blank copolymers, and Rsv-NFs for 24,36 , and $48 \mathrm{~h}$, respectively. The concentration of Rsv was set to $2,4,8,16$, and $32 \mu \mathrm{M}$, while Rsv-NFs were administered at the equivalent dose of free Rsv. After the incubation, $50 \mu \mathrm{L}$ XTT solution was added to each well of the plate and incubated for another $18 \mathrm{~h}$ at $37^{\circ} \mathrm{C}$ in the incubator. Then the plates were read at a wavelength of $490 \mathrm{~nm}$ with $670 \mathrm{~nm}$ as a reference.

2.2.7. Edu Staining and AO/EB Dual Staining. U87 cells were treated with Rsv and Rsv-NFs at an equivalent dose of $\mu \mathrm{M}$ for $36 \mathrm{~h}$ and then washed out with PBS. $300 \mu \mathrm{L} 50 \mu \mathrm{M}$ Edu solution was added to the wells for an incubation time of $2 \mathrm{~h}$, followed by a staining of Hoechst 33342 for $5 \mathrm{~min}$ in the dark. After washing, air-drying, and fixing with $90 \%$ glycerol, cells were observed under a fluorescence microscope with an excitation wavelength of $350 \mathrm{~nm}$ and an emission wavelength 
of $550 \mathrm{~nm}$. Cells stained with Edu were counted from five different fields.

U87 cells were treated with Rsv and Rsv-NFs at an equivalent dose of $\mu \mathrm{M}$ for $36 \mathrm{~h}$ and then washed out with PBS. Cells were then stained by dual fluorescent staining solution $(1 \mu \mathrm{L})$ containing $100 \mu \mathrm{g} / \mathrm{mL}$ AO and $100 \mu \mathrm{g} / \mathrm{mL}$ EB (AO/EB, Sigma, St. Louis, MO). Cells were immediately observed under a fluorescence microscope.

2.2.8. Clonogenic Assay. U87 cells were seeded in T 25 flasks and allowed to adhere to $80-90 \%$ confluent before drug treatment. After being treated with Rsv or Rsv-NFs at a series of equivalent doses $(3,6$, and $12 \mu \mathrm{M})$ for $36 \mathrm{~h}$, cells were trypsinized and seeded in 6-well plate at different density. After 10 days, cells were stained with $0.5 \%$ crystal violet for $2 \mathrm{mins}$, washed out, and air-dried in room temperature. The colonies at each well were counted. Plate efficiency (PE) and survival fraction (SF) were calculated with the following equations:

$$
\begin{aligned}
& \mathrm{PE}=\frac{\text { number of colonies counted }}{\text { number of cells plated }} * 100 \%, \\
& \mathrm{SF}=\frac{\mathrm{PE} \text { of treated sample }}{\mathrm{PE} \text { of control }} * 100 \% .
\end{aligned}
$$

2.2.9. Wound Healing Assay. Cellular migration ability was evaluated through the wound healing assay. Briefly, U87 cells were seeded in 6-well plates and allowed to grow till confluent monolayers were observed. A vertical clear space "wound" was made by a sterile pipette tip. The time that the wound was made was designated as $0 \mathrm{~h}$ and the margins of the wound were photographed by microscopy. After cells were incubated with Rsv and Rsv-NFs at the equivalent doses for $36 \mathrm{~h}$, the same fields of the wound margin were screened again. Adobe Photoshop was applied to superpose the pictures and wound area was measured by Scion Image Analysis Software (Scion Corporation, Frederick, MD, USA). Wound healing rate was calculated by the following equation: wound healing rate = (the average area of wound at $0 \mathrm{~h}$ - the average area of wound at $36 \mathrm{~h}$ )/the average area of wound at $0 \mathrm{~h}$.

2.2.10. Transwell Assay. After growing to subconfluency, U87 cells were cultured in nonserum medium for $24 \mathrm{~h}$. Cells were then trypsinized and resuspended in serum-free medium. After $2 * 10^{4}$ cells were seeded in the upper chamber, the equivalent doses of Rsv and Rsv-NFs were incubated with the cells for $36 \mathrm{~h}$. The invaded cells on the lower membrane surface were then fixed with methanol and stained with $0.1 \%$ crystal violet. The cells were photographed and counted.

2.2.11. Protein Expression. Protein expression was examined by western blot as described in previous studies [21]. Whole cell lysates were fractionated by SDS-PAGE and then transferred into nitrocellulose membranes. After being blocked by $5 \%$ nonfat milk, the membranes were incubated overnight with primary antibodies including antiphospho-ERK, antiERK, anti-Bcl-1, anti-Bcl-xl, and anti-Bax. The blots were incubated with secondary antibodies according to the origin
TABLE 1: Characterization of the synthesized mPEG-PCL copolymers.

\begin{tabular}{lcccc}
\hline Copolymer & $\mathrm{Mn}^{1}$ & $\mathrm{Mn}^{2}$ & $\mathrm{Mw}^{2}$ & $\mathrm{Pd}\left(\mathrm{Mw}^{2} / \mathrm{Mn}^{2}\right)$ \\
\hline mPEG4k-PCL20k & 25160 & 21760 & 38480 & 1.76
\end{tabular}

Pd: polydispersity, defined as the ratio of weight-average molecular weight to the number-average molecular weight $(\mathrm{Mw} / \mathrm{Mn})$.

${ }^{1}$ Determined by ${ }^{1} \mathrm{H}$ NMR.

${ }^{2}$ Determined by GPC.

of primary antibodies, respectively, and then activated by an ECL kit (GE Healthcare, Piscataway, NJ).

2.2.12. In Vivo Study. Male nude mice were raised under specific pathogen-free circumstances. The experiments were in compliance with animal guidelines approved by the Animal Care Committee of Nanjing Medical University. $0.05 \mathrm{~mL}$ cell suspension containing 1 million U87 cells was injected into the left axillary space of the mice. After 7 to 10 days, the mice with tumor volume of around $50 \mathrm{~mm}^{3}$ were selected and this day was designated as Day 1 . Mice were divided to four groups with each group containing 6 mice and treated with blank NFs, free Rsv, and Rsv-NFs, respectively. The dose of Rsv was at an equivalent dose of $10 \mathrm{mg} / \mathrm{kg}$. Blank NFs and RsvNFs were implanted on the tumor nodule through a small surgery while free Rsv was injected intratumorally at the same dose. Mice were ear tagged and tumors were measured by an electronic caliber every other day. Tumor volume was calculated by the formula $\left(W^{2} * L\right) / 2$, where $W$ is the tumor measurement at the widest point and $L$ is the tumor dimension at the longest point.

2.2.13. Statistics. Data were presented as the mean \pm SD of three independent experiments. Statistical analysis for the comparison of relative groups was based on Student's $t$-test or one-way ANOVA analysis with SPSS software. Significance was accepted at the 0.05 level of probability.

\section{Results and Discussion}

3.1. Synthesis and Characterization of MPEG-PCL Copolymers. The obtained mPEG-PCL copolymers were characterized by ${ }^{1} \mathrm{H}$ NMR and GPC. The calculated molecular weight was listed in Table 1 . The purity of the polymer was demonstrated by the only peak of mPEG-PCL copolymer in GPC curve (see Figure S1 of the Supplementary Material available online at http://dx.doi.org/10.1155/2016/5918462). The molecular weight and molecular weight distribution of mPEGPCL from GPC were also listed in Table 1. As shown in Table 1, the molecular weight from NMR and GPC was consistent with the feeding ratios. In addition, the number of molecular weight from GPC was lower than that from NMR, which meant that GPC data were relative values based on polystyrene standards [22].

3.2. Preparation and Characterization of Rsv-NFs. As shown in Figure S2, SEM was performed to observe Rsv-NFs with a uniformed size of about a hundred nanometer (Figure S2). FTIR spectrum in Figure S3 showed three characteristic 

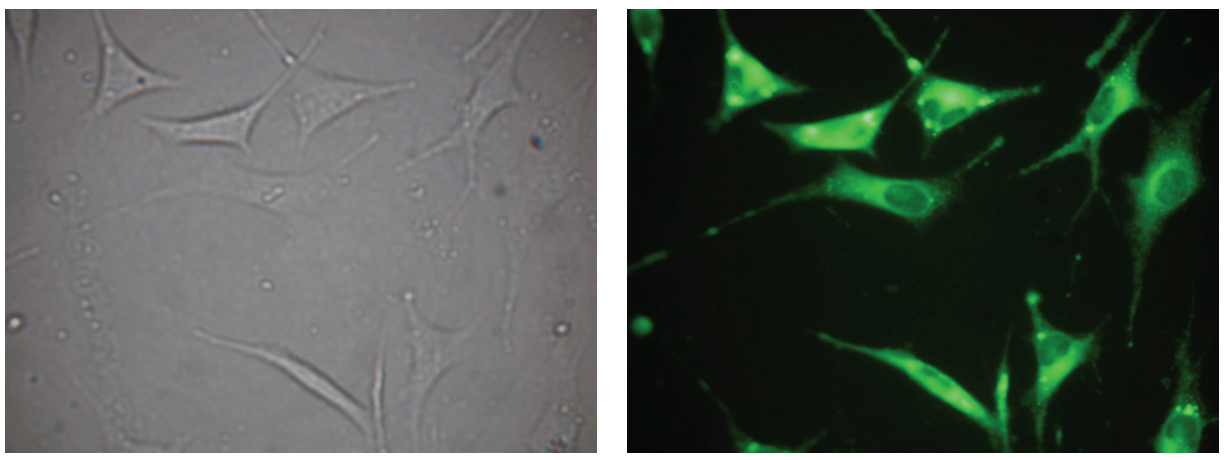

(a)

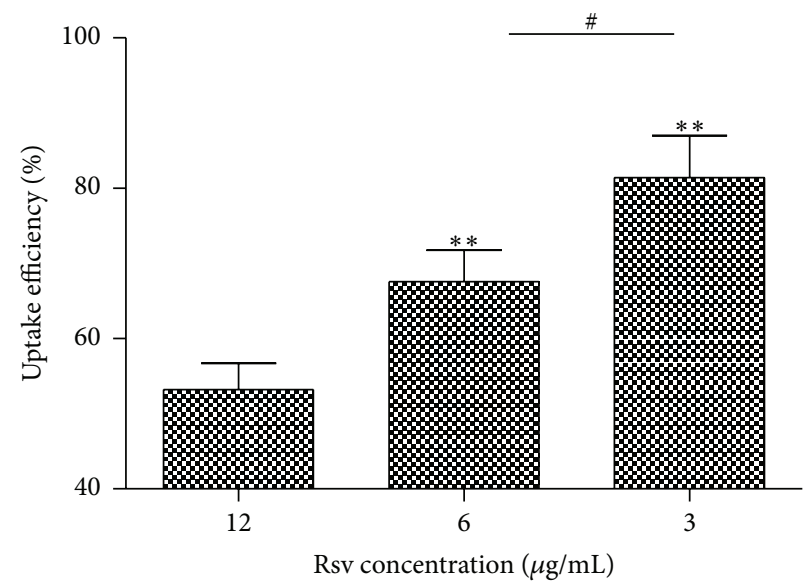

(b)

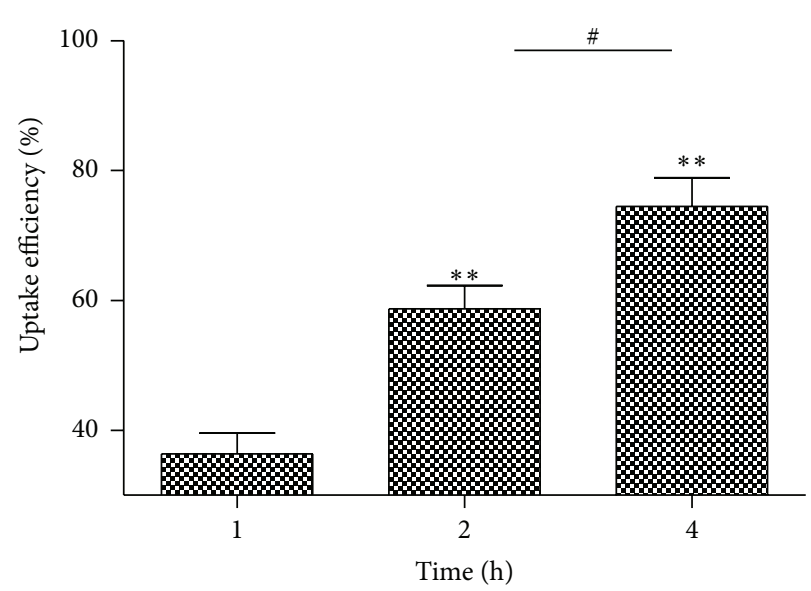

(c)

FIGURE 1: Cellular uptake of Rsv-NFs by U87 cells. (a) Pictures of U87 cells incubated with coumarin-6 loaded Rsv-NFs. (b) Uptake efficiency of Rsv-NFs by U87 cells with different concentrations. (c) Uptake efficiency of Rsv-NFs by U87 cells with different incubation periods. In Figure 1(b), ** means $p<0.01$ versus the group of $12 \mu \mathrm{M}$ Rsv. In Figure $1(\mathrm{c}), * *$ means $p<0.01$ versus the group of $1 \mathrm{~h}$ incubation. \# means $p<0.05$ versus the corresponding group.

peaks of pure Rsv, which corresponded to the three typical strong bands at 1383.85 (C-O stretching), 1586.53 (C-C olefinic stretching), and $1606.21 \mathrm{~cm}^{-1}$ (C-C aromatic doublebond stretching) [23]. As reported in previous study, PCL was characterized by the two bands corresponding to amorphous $\left(1735 \mathrm{~cm}^{-1}\right)$ and crystalline $\left(1725 \mathrm{~cm}^{-1}\right)$ absorption $[24,25]$. In the FTIR spectrum of mPEG-PCL, there was a strong absorption at $1728 \mathrm{~cm}^{-1}$, indicating the high PCL crystallinity inside the mPEG-PCL nanofibers. As reported earlier, PCL crystallinity was one of the main factors that affect the drug loading efficiency. There was a blue shift of the $\mathrm{C}=\mathrm{O}$ vibration from $1728 \mathrm{~cm}^{-1}$ to $1730 \mathrm{~cm}^{-1}$ in the spectrum of Rsv-NFs. Possible explanations may be some form of interaction between Rsv and the $\mathrm{C}=\mathrm{O}$ group of $\mathrm{mPEG}-\mathrm{PCL}$. Moreover, the characteristic peaks of Rsv and mPEG-PCL in the spectrum of Rsv-NFs demonstrated the successful loading of Rsv into Rsv-NFs.

3.3. In Vitro Release Study. Figure S4 showed the in vitro release pattern of two kinds of Rsv-NFs. For the lower loading nanofibers (5 wt\%), there was an initial burst of more than $20 \%$ drugs in the first several hours, followed by a relatively controlled release in the rest of hours. At the end of the experiment $(128 \mathrm{~h})$, nearly $60 \%$ of the loading drug was released from the nanofibers. In contrast, a much faster release pattern was observed in higher loading Rsv-NFs at $15 \mathrm{wt} \%$. The different release kinetics suggested that drug loading efficiency had a great impact on the release pattern of the nanofibers. According to the structure of the drug loaded nanofibers, the hydrophobic PCL of the nanofiber functioned as the main part to encapsulate poor soluble Rsv. As the drug loading content increased without the corresponding augment of the amount of PCL, the interaction of the hydrophobic PCL and Rsv weakened to some extent, which led to the faster release of Rsv-NFs. Moreover, during the preparation process, some of the drug was finally entrapped inside the nanofibers and some drug was absorbed to the surface of the nanofibers. Therefore, high drug loading might cause the relatively high drug absorption to the surface, thereby leading to the faster burst release of the drug loaded nanofibers. Similar results were also reported by a previous study about Curcumin loaded nanofibers [26].

3.4. Cellular Uptake of Rsv-NFs. As reported previously, the uptake efficiency of loaded drugs was primarily influenced by 


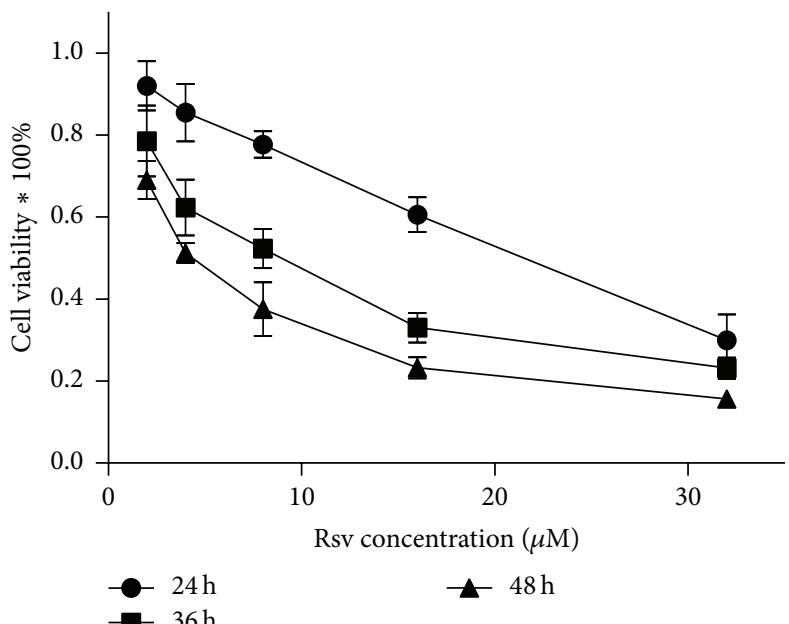

(a)

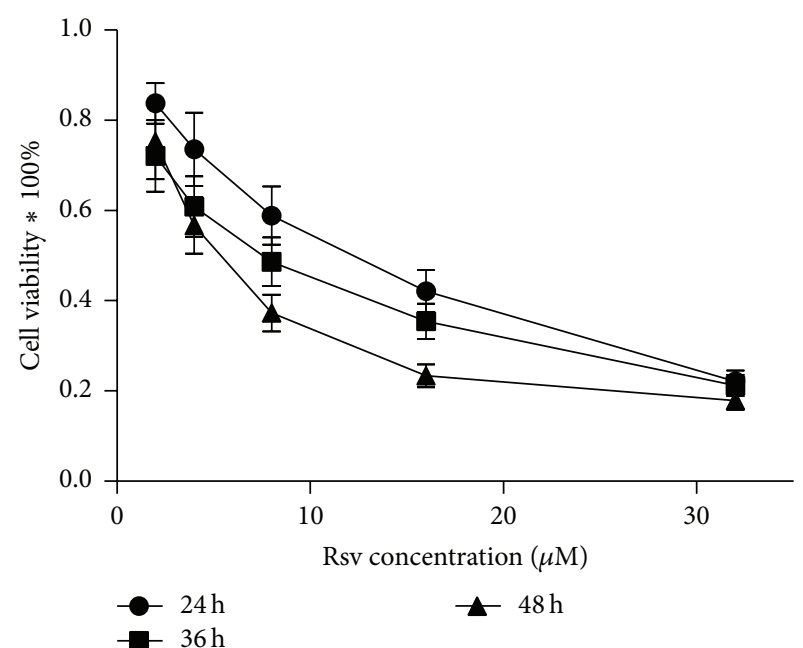

(b)

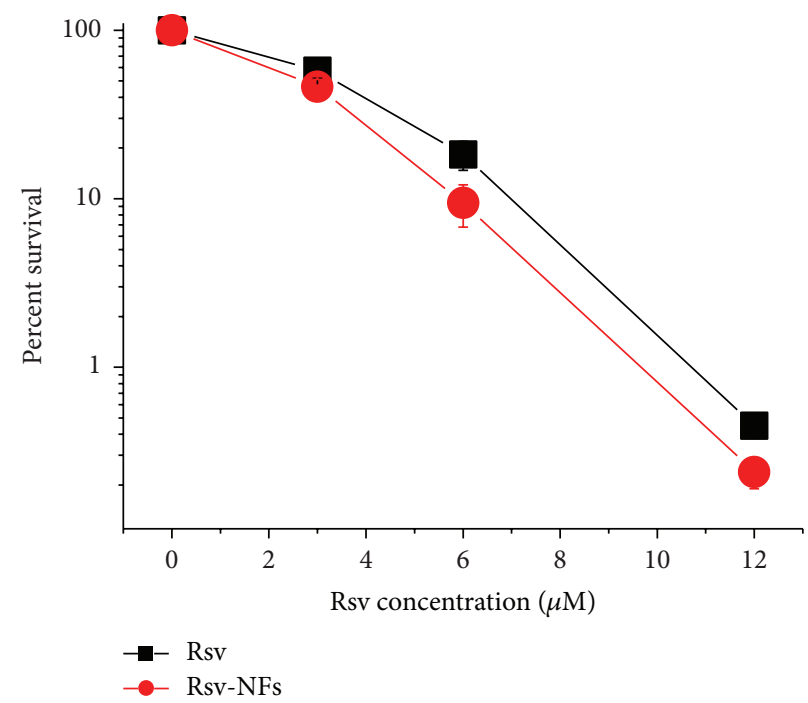

(c)

FIGURE 2: In vitro antitumor effect of Rsv-NFs. (a) Cytotoxicity of Rsv-NFs against U87 cells for 48 hr. (b) Cytotoxicity of Rsv against U87 cells for $48 \mathrm{hr}$. (c) Clonogenic assay of Rsv and Rsv-NFs in U87 cells.

the physicochemical characteristics of nanocarriers instead of the drug since the drug is mostly encapsulated inside the nanocarriers [21]. In the current study, coumarin-6 was utilized as fluorescent marker to trace the nanofibers. Twohour incubation with the cells was enough for the fluorescent Rsv-NFs to enter the cells (Figure 1). From the fluorescent photos, Rsv-NFs were mainly located in the cytoplasm instead of being in the nucleus. The cellular uptake efficiency of nanofibers was closely related to the drug loading content.

As shown in Figures 1(b) and 1(c), higher loading efficiency of coumarin-6 led to brighter fluorescence in cells, which means higher uptake efficiency. As reported in previous studies, cellular uptake of nanofiber was mainly through the absorption mediated endocytosis [1]. However, the internalization of free coumarin- 6 was dependent on diffusion with most of the coumarin- 6 trapped in cell membrane.
Therefore, Rsv-NFs could transport the lipophilic drug into the cells through efficient endocytosis.

3.5. In Vitro Cytotoxicity and Apoptotic Induction of Rsv-NFs. As shown in Figures 2(a) and 2(b), both Rsv and Rsv-NFs had a time- and dose-dependent cytotoxicity against U87 cells. When cells were incubated with both drugs for $24 \mathrm{~h}$, free Rsv showed a stronger cell growth inhibitory effect than Rsv-NFs did at the equivalent dose. As the incubation time increased to $36 \mathrm{~h}$, both Rsv-NFs and Rsv led to similar toxicity to cells at the equivalent concentration. Moreover, further extension of incubation time to $48 \mathrm{~h}$ resulted in a faster increase in the cytotoxicity of Rsv-NFs than that of free Rsv. For example, about $85 \%$ cells survived after $24 \mathrm{~h}$ incubation with $4 \mu \mathrm{M}$ Rsv while only $73 \%$ cells were alive when incubated with the same dose of Rsv-NFs. However, after $72 \mathrm{~h}$ incubation, there 

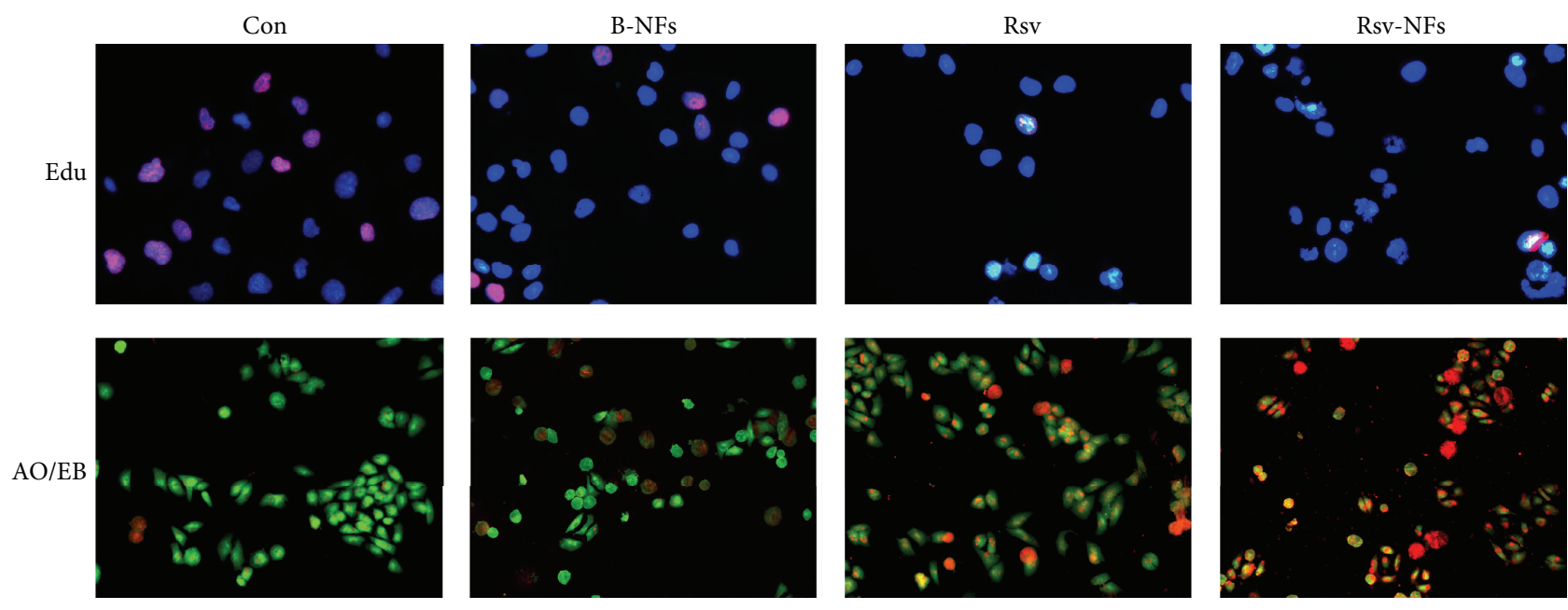

(a)

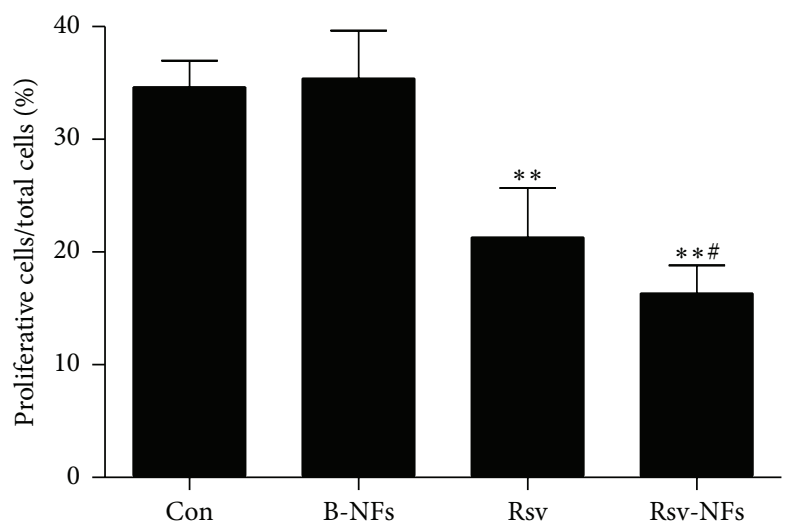

(b)

Figure 3: Apoptotic induction of Rsv-NFs and Rsv in U87 cells. The upper panel of (a): Edu/Hoechst 33342 dual staining; the lower panel of (a): AO/EB dual staining. Bar graph (b): quantitative analysis of apoptosis rate by FACS. $* *$ represents $p<0.01$ versus control group. \# represents $p<0.05$ versus the corresponding group.

were only $51 \%$ cells alive in Rsv-NFs treated group while the viability was more than $56 \%$ in the groups of Rsv treatment. In addition, Table 1 indicated that the $\mathrm{IC}_{50}$ value of Rsv-NFs for $24 \mathrm{~h}$ was higher and decreased faster than that of Rsv as incubated time extended.

For proliferation detection, cells were dually stained by Edu and Hoechst 33342 (Figure 3). Red staining from Edu stood for proliferative cells with blue staining by Hoechst 33342 indicating cell nucleus. From Figure 3, when cells were incubated with the equivalent dose of Rsv and Rsv-NFs, there was less red fluorescence in Rsv-NFs treated group than in the group of Rsv, which demonstrated the stronger cytotoxicity of Rsv-NFs.

Moreover, AO/EB dual staining was applied to visualize the apoptosis and necrosis of cells. AO can penetrate the intact membrane of living cells and early apoptotic cells and stain them green while EB can only enter late apoptotic cells and dead cells with broken membrane and stain them red. As shown in the lower panel of Figure 3(a), cells underwent obvious apoptosis and necrosis when treated with either Rsv or Rsv-NFs. Most importantly, Rsv-NFs led to more early apoptosis and late apoptosis (necrosis) of U87 cells than the equivalent dose of Rsv did.

Previous studies have reported the preparation of drug loaded nanofibers made by electrospinning [17, 18, 27]. Most of them focused on the characterization of nanofibers [18]. Few of them evaluate the antitumor effect systematically. For example, Zupančič et al. successfully constructed Rsv-loaded nanofibers with PCL as drug carriers. They characterized the nanofiber by FTIR, DSC, and so forth with no further evaluation on the anticancer effect [28]. Here we studied the in vitro antitumor effect of Rsv-NFs through XTT assay, proliferation and apoptosis staining, and clonogenic assay. XTT assay and cell staining indicated that Rsv-NFs were more cytotoxic than free Rsv against cells within certain range of concentrations, which was in accordance with the results from Edu staining that less proliferation was observed in the Rsv-NFs treated group.

3.6. Clonogenic Assay. As indicated in previous studies, there are two ways to evaluate the cell drug sensitivity. One way is to measure the cytotoxicity, which assesses the cellular 

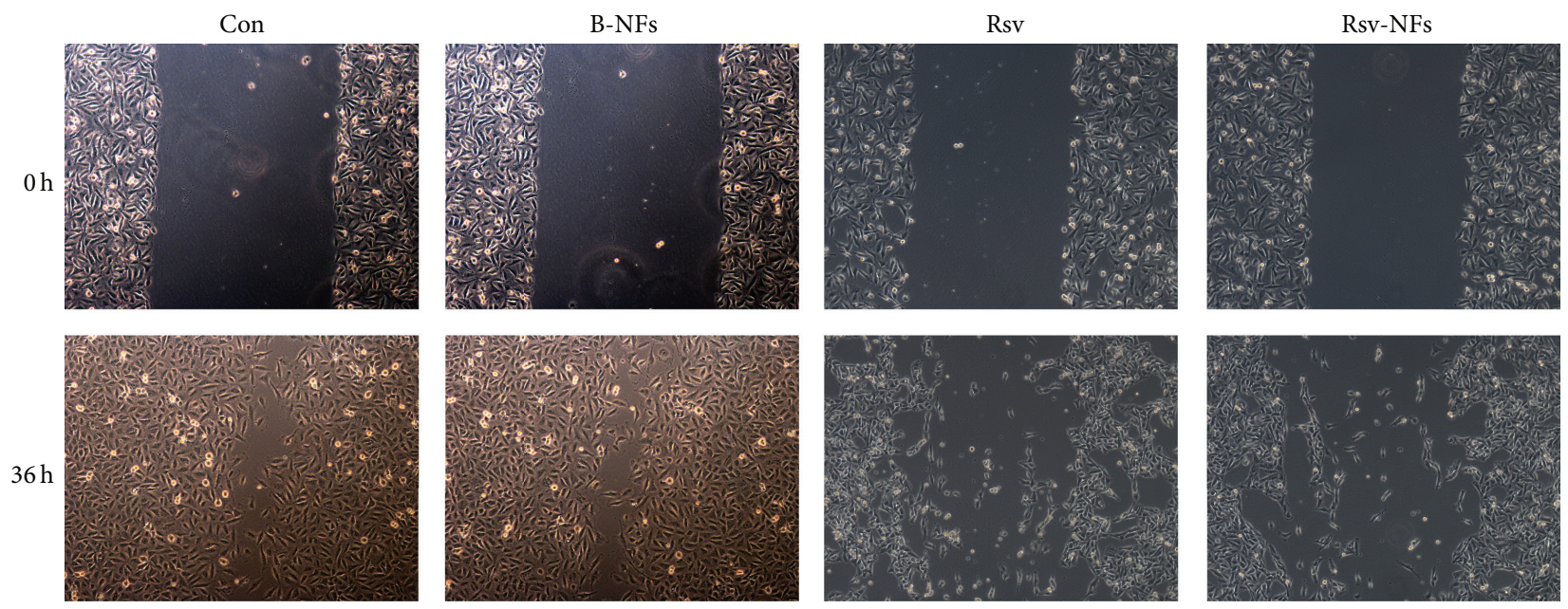

(a)

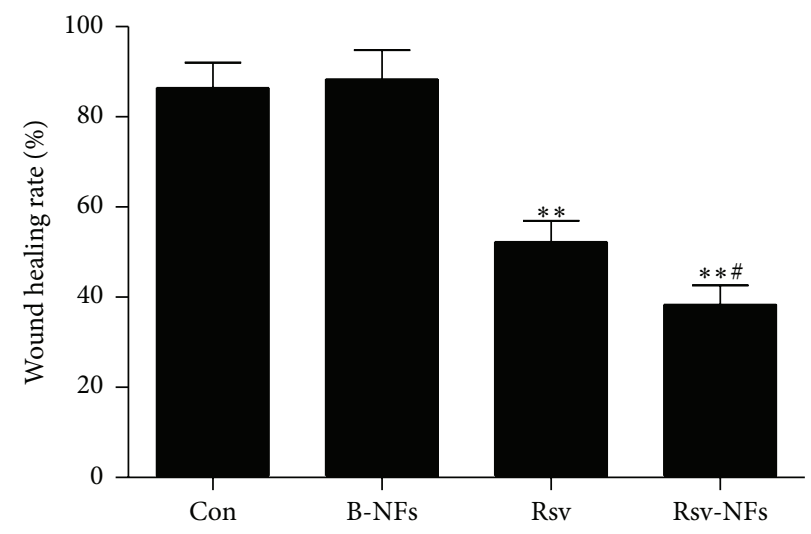

(b)

FIGURE 4: Wound healing assay of U87 cells when exposed to the equivalent dose of either Rsv or Rsv-NFs. $* *$ represents $p<0.01$ versus control group. \# represents $p<0.05$ versus the corresponding group.

ability of proliferation after drug treatment. The other way is to study the cell survival by clonogenic assay to evaluate the ability of individual cells to form colonies [29]. Cells that are able to go through mitosis but cannot divide and reproduce a big number of progeny are considered as dead due to the loss of reproductive integrity. Only cells that possess the ability to proliferate and reproduce a large colony are regarded as "clonogenic" [30]. Therefore, cell survival cure generated from clonogenic assay describes a relationship between cytotoxic drug and the percent of cells that survive.

In the current study, clonogenic assay was performed to evaluate the ability of proliferation and clonogenesis of glioma cells. Figure 2(c) showed that the survival curve of cells exposed to either Rsv or Rsv-NFs. At the three dose levels, cells treated with Rsv-NFs exhibited less clonogenic abilities than cells exposed to the equivalent dose of Rsv. The numbers of cell colonies in the Rsv-NFs treated groups were significantly lower than those in corresponding Rsv-treated groups, which means that the clonogenic ability of cells treated with Rsv-NFs was substantially abrogated compared to free Rsv-treated cells.
3.7. Inhibition on the Migration and Invasion of Cells by RsvNFs. There are several important steps during the process of tumor metastasis including the ECM degradation, vascular penetration, and adhesion elsewhere [31]. During the whole process, the ability of cells to migrate and invade is the main factor that contributes to the tumor metastasis [32].

Here we employed a highly invasive cell line to evaluate the migration and invasion of cells when exposed to Rsv and Rsv-NFs by wound healing and transwell assay. As shown in Figure 4, both Rsv-NFs and Rsv inhibited the migration of cells in a dose-dependent manner. Moreover, Rsv-NFs delayed the migration of cells more efficiently than free Rsv did at the equivalent concentration. Similar trends could be observed from the results of transwell assay (Figure 5). The number of successful invading cells in the Rsv-NFs treated group was significantly lower than that in Rsv treated group, which demonstrated an enhanced inhibitory effect of RsvNFs.

3.8. Protein Expression. Protein expressions were evaluated by western blot to elucidate the possible mechanism of 

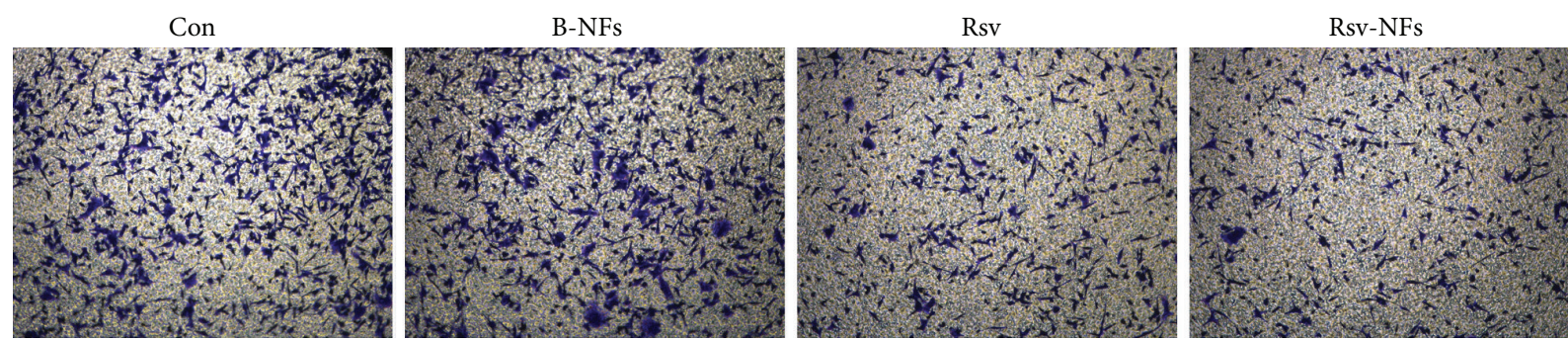

(a)

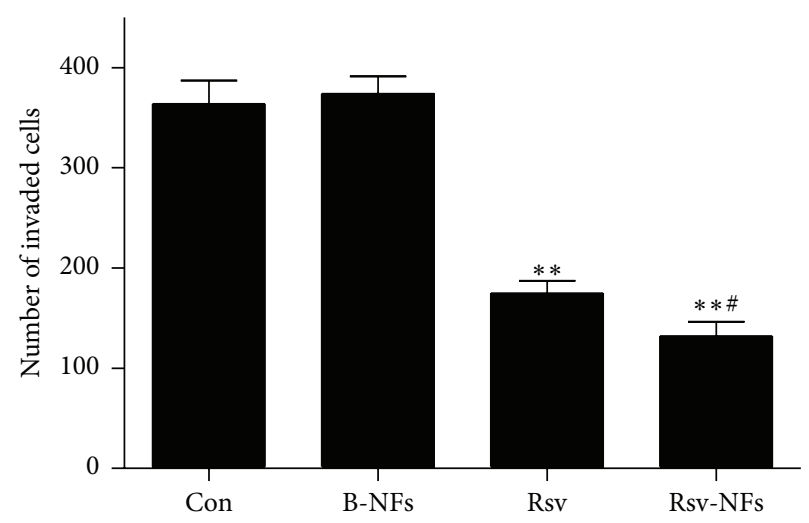

(b)

FIGURE 5: Transwell assay of U87 cells when exposed to the equivalent dose of either Rsv or Rsv-NFs. $* *$ represents $p<0.01$ versus control group. \# represents $p<0.05$ versus the corresponding group.

antitumor effects of Res-NFs. As a member of the mitogenactivated protein kinase (MAPK) family, extracellular signalregulated kinase (ERK) is constitutively expressed and contributes to cell proliferation and survival in a series of cancer cells [33]. The close relationship between ERK and apoptotic proteins has also been established in previous studies. The activity of ERK pathway has been proved with the upregulation of proapoptotic members of the Bcl-2 family, such as Bax and Bak, as well as the downregulation of antiapoptotic members, such as Bcl-2 and Bcl-xl $[34,35]$.

It is shown in Figure 6 that both Res and Res-NFs inhibited the expression of $\mathrm{p}$-ERK when compared to control. Most importantly, the expression of p-ERK in cells treated with Res-NFs was significantly lower than that treated with the equivalent dose of Res. Moreover, Res-NFs were more efficient to induce proapoptotic protein Bax and decrease the antiapoptotic proteins Bcl-2 and Bcl-xl (Figure 6). Therefore, it is demonstrated that Res-NFs induced the apoptosis of U87 cells through the inhibition of ERK activity and the regulation of apoptotic pathway.

3.9. In Vivo Study of Rsv-NFs. Figure 7 shows the curve of tumor volume of tumor bearing mice in different groups. It is noted that both Rsv and Rsv-NFs dose-dependently delayed the growth of tumors with no antitumor effect observed in either control group or blank nanofiber treated group. From Day 9 to the end of observation, the difference between the groups of Rsv and control was significant $(p<0.05)$, as was the difference between the groups of Rsv-NFs and control $(p<0.05)$. Most importantly, the antitumor efficiency of RsvNFs was much more stronger than that of free Rsv as the mean tumor volume of mice treated by Rsv-NFs was significantly smaller than that of mice with the treatment of the equivalent dose of Rsv from Day 11 to the end of experiment $(p<0.01)$. For instance, mice in the group of Rsv reached the standard of euthanization $\left(1.998 \pm 0.098 \mathrm{~cm}^{3}\right)$ while the mice in RsvNFs treated group was only a bit larger than half the size $\left(1.213 \pm 0.087 \mathrm{~cm}^{3}\right)$ of euthanization standard.

Here Rsv-NFs mats were implanted to cover the tumor nodule through a small surgery instead of injection through tail vein. Three advantages could be achieved due to the characteristic pharmacokinetics profile of Rsv-NFs through local implantation of the drug loaded nanofibers. First, a sustained release of Rsv around the tumor through local implantation of nanofiber means a continuous exposure of tumor nodule to the drug. Second, controlled release of Rsv around the tumor will increase the penetration of drug into the tumor since the density of neovessels on the surface of tumor mass is much more higher than that inside the tumor. This will lead to an effective long-term high drug concentration in the tumor site, thereby generating stronger antitumor effect. Third, the efficient cellular uptake of Rsv-NFs through endocytosis also contributes to the increase of intracellular drug concentration. Two previous reports which studied the in vivo efficiency of drug loaded nanofibers also supported the idea that local implantation increases the antitumor effect of drug loaded nanofibers $[36,37]$. 


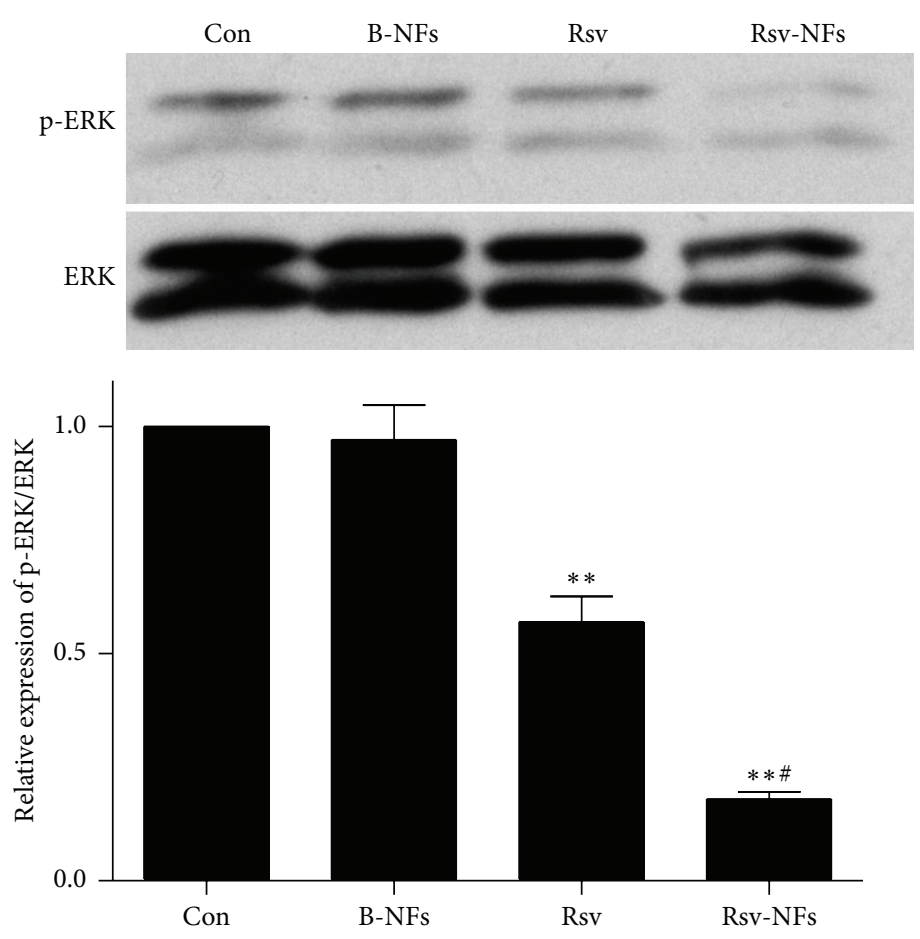

(a)

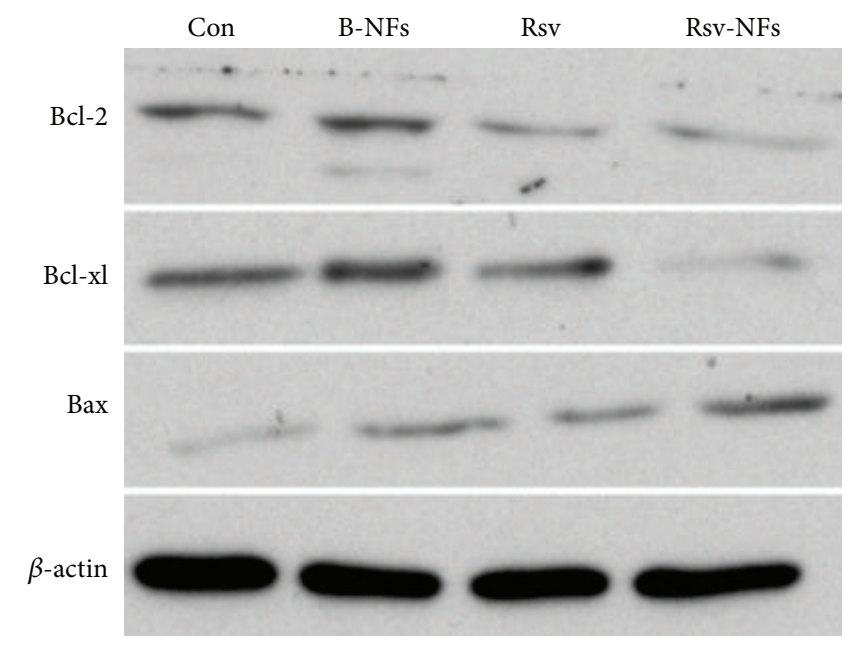

(b)

FIGURE 6: Protein expression in U87 cells exposed to different treatment. (a) Protein expression of p-ERK/ERK with semiquantitative analysis. (b) Expression of apoptotic proteins in cells. $* *$ means $p<0.01$ versus control group. \# means $p<0.05$ versus the Rsv group.

\section{Conclusion}

In the current study, we prepared Rsv-loaded nanofibers by electrospinning with mEPG-PCL as drug carriers. Characterization studies showed the successful encapsulation of Rsv in the nanofibers with an in vitro sustained release pattern. In vitro XTT assay suggested the superior cytotoxicity of RsvNFs with more apoptosis induction. Cells that exposed to Rsv-NFs showed less clonogenic ability when compared to the equivalent dose of free Rsv. In addition, the migration and invasion ability of cells were also significantly lower when treated with Rsv-NFs. In the in vivo study, local implantation of Rsv-NFs greatly increased the growth inhibitory effect compared to free Rsv. Therefore, results from the current study demonstrated a promising way to improve the antitumor effect of Rsv by nanofiber delivery.

\section{Competing Interests}

The author reports no conflict of interests in this work.

\section{Authors' Contributions}

Hui Zhou, Xiaoxing Liu, and Fenglei Wu contributed equally to the study. 


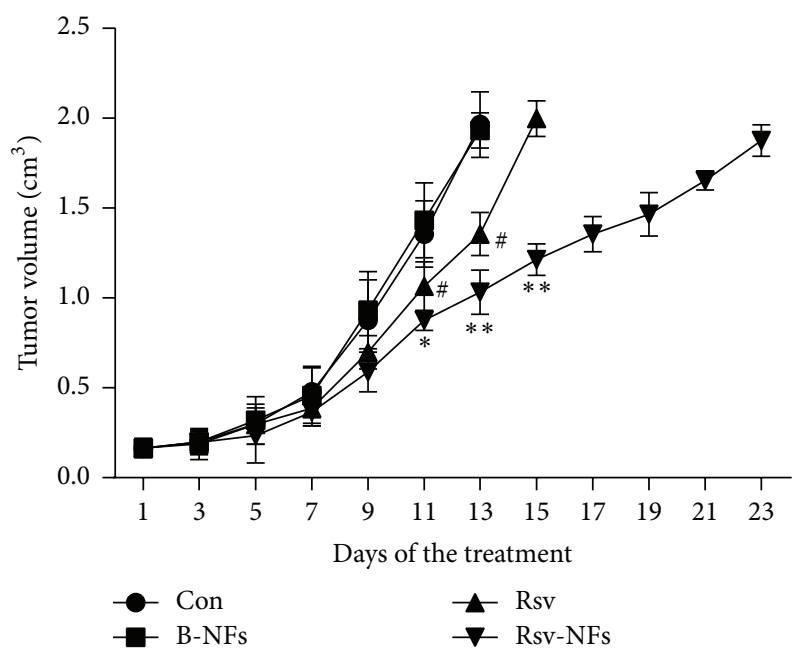

(a)

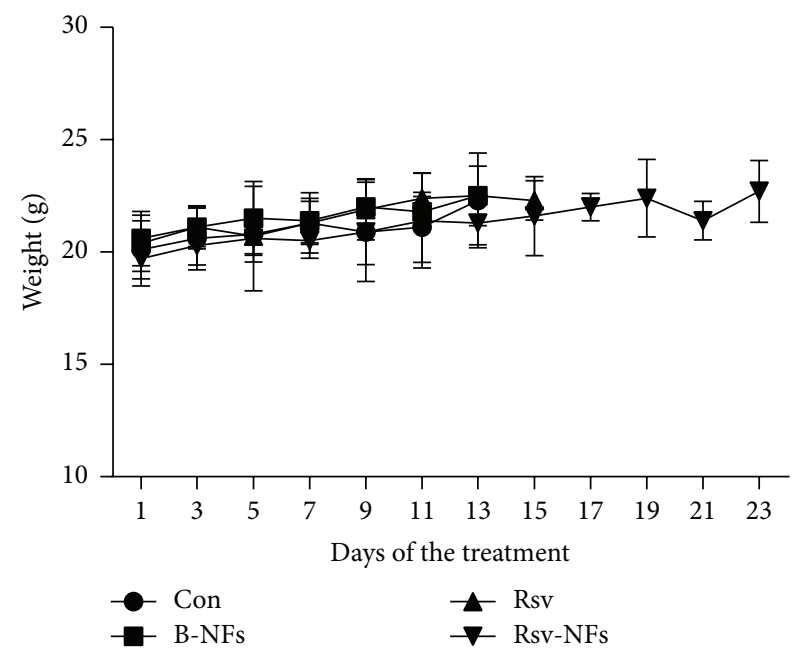

(b)

FIGURE 7: In vivo antitumor effect of Rsv-NFs in a xenograft of U87 cells. (a) Growth curve of tumor volume from different groups of mice. (b) Change of weight of the mice in different groups. \# represents $p<0.05$ versus control group. * represents $p<0.05$ versus the group treated by Rsv. $* *$ represents $p<0.01$ versus the group treated by Rsv.

\section{Acknowledgments}

This work was supported by the National Natural Science Foundation of China (no. 81401968) and Natural Science Foundation of Jiangsu Province (BK20151157 and BK20141245).

\section{References}

[1] X. Li, D. Zhen, X. Lu et al., "Enhanced cytotoxicity and activation of ROS-dependent c-Jun NH 2-terminal kinase and caspase- 3 by low doses of tetrandrine-loaded nanoparticles in Lovo cells-a possible Trojan strategy against cancer," European Journal of Pharmaceutics and Biopharmaceutics, vol. 75, no. 3, pp. 334-340, 2010.

[2] X. Lu, H. Xu, B. Sun, Z. Zhu, D. Zheng, and X. Li, "Enhanced neuroprotective effects of resveratrol delivered by nanoparticles on hydrogen peroxide-induced oxidative stress in rat cortical cell culture," Molecular Pharmaceutics, vol. 10, no. 5, pp. 20452053, 2013.

[3] H. Zhang, K. Sun, J. Ding et al., "Harmine induces apoptosis and inhibits tumor cell proliferation, migration and invasion through down-regulation of cyclooxygenase- 2 expression in gastric cancer," Phytomedicine, vol. 21, no. 3, pp. 348-355, 2014.

[4] X. Lu, C. Ji, H. Xu et al., "Resveratrol-loaded polymeric micelles protect cells from $\mathrm{A} \beta$-induced oxidative stress," International Journal of Pharmaceutics, vol. 375, no. 1-2, pp. 89-96, 2009.

[5] H. Zhang, D. Zheng, J. Ding, H. Xu, X. Li, and W. Sun, "Efficient delivery of ursolic acid by poly(N-vinylpyrrolidone)-block-poly ( $\varepsilon$-caprolactone) nanoparticles for inhibiting the growth of hepatocellular carcinoma in vitro and in vivo," International Journal of Nanomedicine, vol. 10, pp. 1909-1920, 2015.

[6] H. Zhang, X. Li, J. Ding et al., "Delivery of ursolic acid (UA) in polymeric nanoparticles effectively promotes the apoptosis of gastric cancer cells through enhanced inhibition of cyclooxygenase 2 (COX-2)," International Journal of Pharmaceutics, vol. 441, no. 1-2, pp. 261-268, 2013.
[7] J. Shao, D. Zheng, Z. Jiang et al., "Curcumin delivery by methoxy polyethylene glycol-poly(caprolactone) nanoparticles inhibits the growth of C6 glioma cells," Acta Biochimica et Biophysica Sinica, vol. 43, no. 4, pp. 267-274, 2011.

[8] C. Buhrmann, P. Shayan, P. Kraehe, B. Popper, A. Goel, and M. Shakibaei, "Resveratrol induces chemosensitization to 5fluorouracil through up-regulation of intercellular junctions, epithelial-to-mesenchymal transition and apoptosis in colorectal cancer," Biochemical Pharmacology, vol. 98, no. 1, pp. 51-58, 2015.

[9] O. Holian, S. Wahid, M. J. Atten, and B. M. Attar, "Inhibition of gastric cancer cell proliferation by resveratrol: role of nitric oxide," American Journal of Physiology-Gastrointestinal and Liver Physiology, vol. 282, no. 5, pp. G809-G816, 2002.

[10] Z. Wu, B. Liu, E. Cailing et al., "Resveratrol inhibits the proliferation of human melanoma cells by inducing G1/S cell cycle arrest and apoptosis," Molecular Medicine Reports, vol. 11, no. 1, pp. 400-404, 2015.

[11] P.-C. Hsiao, Y.-E. Chou, P. Tan et al., "Pterostilbene simultaneously induced G0/G1-phase arrest and MAPK-mediated mitochondrial-derived apoptosis in human acute myeloid leukemia cell lines," PLoS ONE, vol. 9, no. 8, Article ID e105342, 2014.

[12] Y.-Z. Liu, K. Wu, J. Huang et al., "The PTEN/PI3K/Akt and $\mathrm{Wnt} / \beta$-catenin signaling pathways are involved in the inhibitory effect of resveratrol on human colon cancer cell proliferation," International Journal of Oncology, vol. 45, no. 1, pp. 104-112, 2014.

[13] A. A. Sprouse and B.-S. Herbert, "Resveratrol augments paclitaxel treatment in MDA-MB-231 and paclitaxel-resistant MDAMB-231 breast cancer cells," Anticancer Research, vol. 34, no. 10, pp. 5363-5374, 2014.

[14] L. Ma, W. Li, R. Wang et al., "Resveratrol enhanced anticancer effects of cisplatin on non-small cell lung cancer cell lines by inducing mitochondrial dysfunction and cell apoptosis," International Journal of Oncology, vol. 47, no. 4, pp. 1460-1468, 2015. 
[15] J. Shao, X. Li, X. Lu et al., "Enhanced growth inhibition effect of resveratrol incorporated into biodegradable nanoparticles against glioma cells is mediated by the induction of intracellular reactive oxygen species levels," Colloids and Surfaces B: Biointerfaces, vol. 72, no. 1, pp. 40-47, 2009.

[16] H. Yin, J. Si, H. Xu et al., "Resveratrol-loaded nanoparticles reduce, oxidative stress induced by radiation or amyloid- $\beta$ in transgenic Caenorhabditis elegans," Journal of Biomedical Nanotechnology, vol. 10, no. 8, pp. 1536-1544, 2014.

[17] M. Sampath, R. Lakra, P. Korrapati, and B. Sengottuvelan, "Curcumin loaded poly (lactic-co-glycolic) acid nanofiber for the treatment of carcinoma," Colloids and Surfaces B: Biointerfaces, vol. 117, pp. 128-134, 2014.

[18] N. Ramalingam, T. S. Natarajan, and S. Rajiv, "Preparation and characterization of electrospun curcumin loaded poly(2hydroxyethyl methacrylate) nanofiber-a biomaterial for multidrug resistant organisms," Journal of Biomedical Materials Research Part A, vol. 103, no. 1, pp. 16-24, 2015.

[19] Y. Geng, P. Dalhaimer, S. Cai et al., "Shape effects of filaments versus spherical particles in flow and drug delivery," Nature Nanotechnology, vol. 2, no. 4, pp. 249-255, 2007.

[20] X. Li, R. Li, X. Qian et al., "Superior antitumor efficiency of cisplatin-loaded nanoparticles by intratumoral delivery with decreased tumor metabolism rate," European Journal of Pharmaceutics and Biopharmaceutics, vol. 70, no. 3, pp. 726-734, 2008.

[21] H. Xu, Z. Hou, H. Zhang et al., "An efficient trojan delivery of tetrandrine by poly(N-vinylpyrrolidone)-block-poly $(\varepsilon$ caprolactone) (PVP-b-PCL) nanoparticles shows enhanced apoptotic induction of lung cancer cells and inhibition of its migration and invasion," International Journal of Nanomedicine, vol. 9, no. 1, pp. 231-242, 2013.

[22] Y. Hu, L. Zhang, Y. Cao, H. Ge, X. Jiang, and C. Yang, "Degradation behavior of poly( $\varepsilon$-caprolactone)- $b$-poly(ethylene glycol)- $b$-poly( $\varepsilon$-caprolactone) micelles in aqueous solution," Biomacromolecules, vol. 5, no. 5, pp. 1756-1762, 2004.

[23] F. Billes, I. Mohammed-Ziegler, H. Mikosch, and E. Tyihák, "Vibrational spectroscopy of resveratrol," Spectrochimica Acta Part A: Molecular and Biomolecular Spectroscopy, vol. 68, no. 3, pp. 669-679, 2007.

[24] H. Danafar, S. Davaran, K. Rostamizadeh, H. Valizadeh, and M. Hamidi, "Biodegradable m-PEG/PCL core-shell micelles: preparation and characterization as a sustained release formulation for curcumin," Advanced Pharmaceutical Bulletin, vol. 4, supplement 2, pp. 501-510, 2014.

[25] X. Li, X. Lu, H. Xu et al., "Paclitaxel/tetrandrine coloaded nanoparticles effectively promote the apoptosis of gastric cancer cells based on 'oxidation therapy', Molecular Pharmaceutics, vol. 9, no. 2, pp. 222-229, 2012.

[26] G. Guo, S. Fu, L. Zhou et al., "Preparation of curcumin loaded poly( $\varepsilon$-caprolactone)-poly(ethylene glycol)-poly( $\varepsilon$ caprolactone) nanofibers and their in vitro antitumor activity against Glioma 9L cells," Nanoscale, vol. 3, no. 9, pp. 3825-3832, 2011.

[27] J. Xie and C.-H. Wang, "Electrospun micro- and nanofibers for sustained delivery of paclitaxel to treat C6 glioma in vitro," Pharmaceutical Research, vol. 23, no. 8, pp. 1817-1826, 2006.

[28] Š. Zupančič, S. Baumgartner, Z. Lavrič, M. Petelin, and J. Kristl, "Local delivery of resveratrol using polycaprolactone nanofibers for treatment of periodontal disease," Journal of Drug Delivery Science and Technology, vol. 30, pp. 408-416, 2015.
[29] V. Bunel, M. Ouedraogo, A. T. Nguyen, C. Stévigny, and P. Duez, "Methods applied to the in vitro primary toxicology testing of natural products: state of the art, strengths, and limits," Planta Medica, vol. 80, no. 14, pp. 1210-1226, 2014.

[30] N. A. P. Franken, H. M. Rodermond, J. Stap, J. Haveman, and C. van Bree, "Clonogenic assay of cells in vitro," Nature Protocols, vol. 1, no. 5, pp. 2315-2319, 2006.

[31] S. A. Äther Enam, M. L. Rosenblum, and K. Edvardsen, "Role of extracellular matrix in tumor invasion: migration of glioma cells along fibronectin-positive mesenchymal cell processes," Neurosurgery, vol. 42, no. 3, pp. 599-608, 1998.

[32] K. Nabeshima, T. Inoue, Y. Shimao, and T. Sameshima, "Matrix metalloproteinases in tumor invasion: role for cell migration," Pathology International, vol. 52, no. 4, pp. 255-264, 2002.

[33] L. O. Murphy and J. Blenis, "MAPK signal specificity: the right place at the right time," Trends in Biochemical Sciences, vol. 31, no. 5, pp. 268-275, 2006.

[34] X. Wang, J. L. Martindale, and N. J. Holbrook, "Requirement for ERK activation in cisplatin-induced apoptosis," The Journal of Biological Chemistry, vol. 275, no. 50, pp. 39435-39443, 2000.

[35] A. Basu and H. Tu, "Activation of ERK during DNA damageinduced apoptosis involves protein kinase $C \delta$," Biochemical and Biophysical Research Communications, vol. 334, no. 4, pp. 10681073, 2005.

[36] H. Singh, E. James, H.-M. Kan, and L. S. Nair, "Fabrication and evaluation of resveratrol loaded polymeric nanofibers," Journal of Biomaterials and Tissue Engineering, vol. 2, no. 3, pp. 228-235, 2012.

[37] M. S. Kamath, S. S. S. J. Ahmed, M. Dhanasekaran, and S. Winkins Santosh, "Polycaprolactone scaffold engineered for sustained release of resveratrol: therapeutic enhancement in bone tissue engineering," International Journal of Nanomedicine, vol. 9, no. 1, pp. 183-195, 2013. 

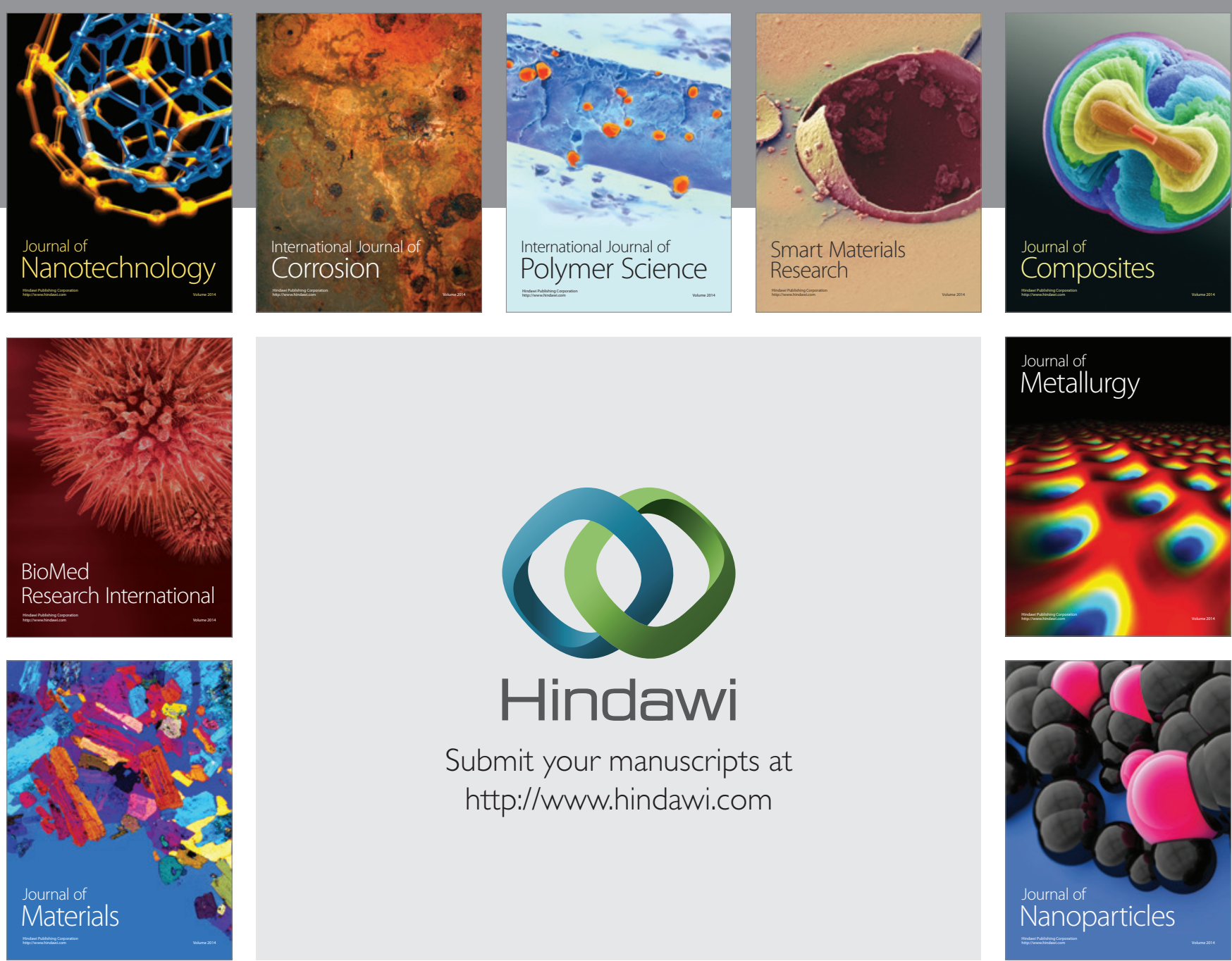

\section{Hindawi}

Submit your manuscripts at

http://www.hindawi.com

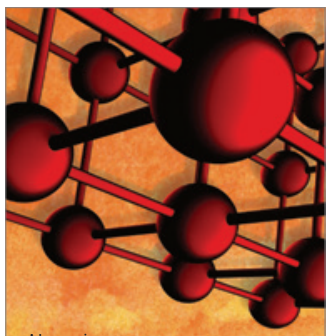

Materials Science and Engineering
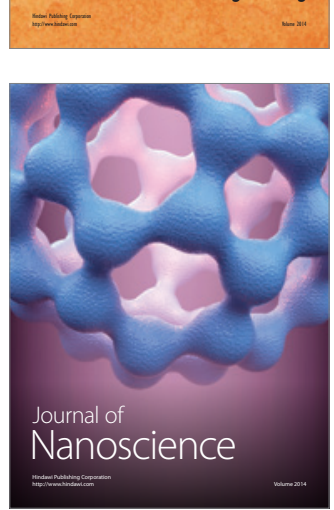
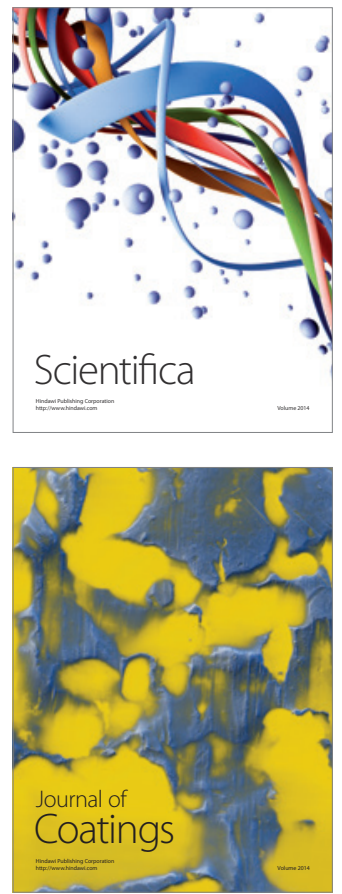
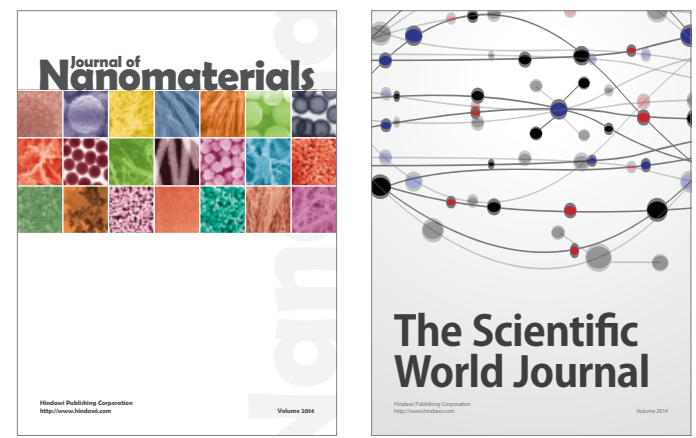

The Scientific World Journal
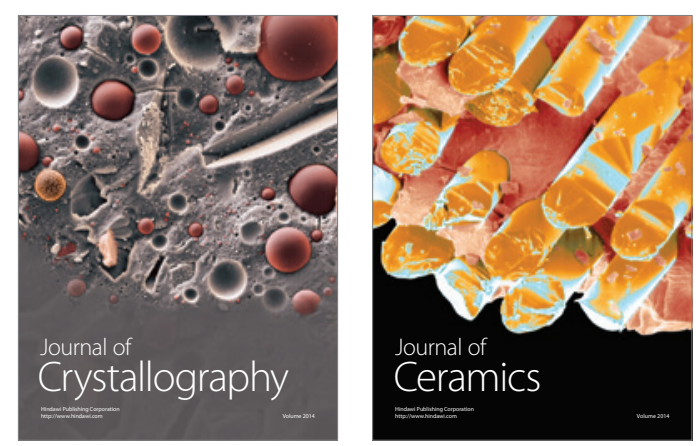
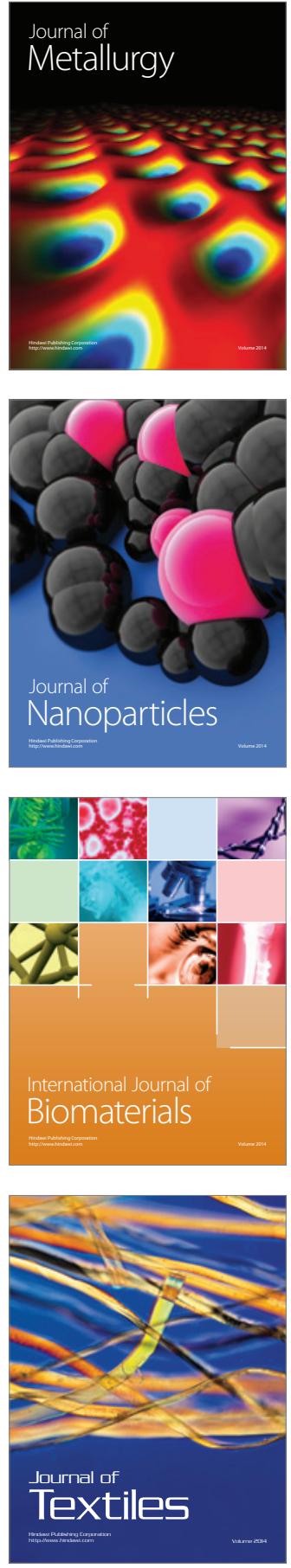